\title{
Pression collective et vulnérabilité individuelle en France : les mariages précoces en milieu immigrant Soninké
}

Yves Zountchégbé Magnon

\author{
(2) OpenEdition \\ Journals \\ Édition électronique \\ URL : https://journals.openedition.org/remi/4213 \\ DOI : $10.4000 /$ remi.4213 \\ ISSN : $1777-5418$ \\ Éditeur \\ Université de Poitiers \\ Édition imprimée \\ Date de publication : 1 décembre 2007 \\ Pagination : 97-108 \\ ISBN : 978-2-911627-47-4 \\ ISSN : 0765-0752
}

Référence électronique

Yves Zountchégbé Magnon, « Pression collective et vulnérabilité individuelle en France : les mariages précoces en milieu immigrant Soninké », Revue européenne des migrations internationales [En ligne], vol. $23-n^{\circ} 3$ | 2007, mis en ligne le 01 décembre 2010, consulté le 15 avril 2022. URL : http:// journals.openedition.org/remi/4213 ; DOI : https://doi.org/10.4000/remi.4213

Ce document a été généré automatiquement le 15 avril 2022.

(c) Université de Poitiers 


\title{
Pression collective et vulnérabilité individuelle en France : les mariages précoces en milieu immigrant Soninké
}

\author{
Yves Zountchégbé Magnon
}

En pays Soninké (Mali, Mauritanie, Sénégal), tout le processus de mise en union des filles est régi par des obligations religieuses et coutumières, et conduit généralement à un âge au mariage précoce. Mais, contrairement à Timera (1996: 123) pour qui les enfants d'immigrants Soninké nés et élevés en France "n'affichent aucune appartenance culturelle africaine dans le comportement quotidien et l'espace civil », nos données suggèrent que le comportement matrimonial des filles reste proche du modèle du pays d'origine en ce qui concerne sa précocité. Cependant, celle-ci, loin d'être le signe de la prééminence absolue de la culture Soninké et de la volonté parentale, est en réalité déterminée par un système de contraintes qui traduit une grande vulnérabilité aussi bien des parents que de leurs filles et, par ricochet, des conjoints de ces dernières. C'est ce que cette note de recherche se propose de montrer à partir d'entretiens réalisés en 2006, dans une commune de la banlieue parisienne, et qui portaient sur la trajectoire matrimoniale de filles mariées jeunes. Nous avons choisi de définir les unions précoces par rapport à l'âge au mariage le plus élevé des filles d'immigrants Soninké dont l'observation est à l'origine de notre étude, c'est-à-dire 21 ans.

Notre terrain a été réalisé auprès d'un échantillon d'une vingtaine d'individus résidant tous en Ile-de-France et appartenant à deux catégories : dix femmes, mariées ou ayant été mariées, nées et élevées en France de parents (père et mère) Soninké ; dix parents immigrés d'origine soninké - dont cinq hommes et cinq femmes - ayant des filles nées et élevées en France et qui sont mariées ou ont été mariées. La vulnérabilité s'analysera, d'une manière générale, en termes d'exposition des individus à des situations de risques sociaux, économiques, juridiques, environnementaux et politiques et de leurs réponses (ou non-réponses) à ces situations (Charbit, 2005). 


\section{Représentations et pratiques relatives au processus matrimonial : des lignes de rupture parents/ filles}

3 Les représentations des filles d'immigrants Soninké nées et élevées en France quant au déroulement d'un processus de mise en union ne sont pas homogènes. Elles varient, si peu soit-il, en fonction de l'histoire personnelle de chaque fille, de sa trajectoire et de ses expériences matrimoniales, de ses conditions de vie, etc. Mais leurs discours sont, en général, plutôt révélateurs de l'influence d'une double socialisation, celle du pays d'origine, essentiellement par le milieu familial, et celle de la société française dans laquelle elles sont nées et ont grandi.

Ce qui apparait en revanche significatif dans la production discursive de nos enquêté(e)s, c'est une orientation différenciée des représentations suivant les générations. En effet, contrairement aux filles, la perception qu'ont les parents, que ce soient les pères ou les mères, quant au processus de mise en union de leur progéniture, reste essentiellement calquée sur le modèle culturel soninké. Les comportements tendent également à suivre la même tendance, se partageant souvent de façon dichotomique, entre discours parentaux et discours des enfants.

5 Nous avons identifié cinq étapes principales du parcours matrimonial où nous avons effectué, comme évoqué ci-dessus, les croisements parents-enfants, d'une part entre représentations, et d'autre part entre représentations et pratiques: (i) relations amoureuses et sexuelles avant le mariage, (ii) union libre/cohabitation, (iii) qualités d'un «bon » mari/mari «idéal», (iv) autonomie financière et matérielle avant le mariage et (v) implication parentale dans le processus de mise en union. Nous nous appuierons, à dessein, sur quelques-unes de ces étapes qui, tout en rendant compte des autres, facilitent la mise en évidence des logiques des acteurs et des situations de vulnérabilité qui en découlent.

\section{Relations amoureuses et sexuelles avant le mariage}

6 Les jeunes filles françaises d'origine Soninké ne font pas du mariage le cadre exclusif d'existence des relations amoureuses et sexuelles. Pour toutes nos enquêtées Soninké de la deuxième génération, les relations amoureuses avec un homme ne doivent pas forcément avoir pour finalité le mariage. Et, à ce propos, elles font bien dans leurs discours, comme toutes les filles françaises de leur âge, une différence entre "petit copain »/ami et mari/époux.

7 En revanche, les avis divergent légèrement lorsqu'on aborde plus spécifiquement la question de la sexualité pré-conjugale. En effet, alors que certaines, évoquant les règles islamiques - l'Islam est la religion de tous nos enquêtés -, pensent que les relations amoureuses avant le mariage devraient se limiter à de simples fréquentations, sans relations sexuelles, d'autres, au contraire, ne conçoivent pas de relations amoureuses sans sexualité. Si le principe de la virginité avant le mariage est perçu comme louable par la plupart d'entre elles, même pour celles qui ne s'y déclarent pas favorables, surtout pour le symbole de la bonne pratique religieuse qu'elle représente et pour la fierté qu'elle donnerait aux parents, elles qualifient en même temps ce principe de 
"dépassé ", "surtout en France où personne ne fait plus ça » et ne le considèrent pas comme un facteur déterminant dans un processus de mise en union.

8 Le ton est unanime chez les parents, que ce soient les pères où les mères, pour expliquer et défendre que le mariage demeure cet acte sacré uniquement en vue duquel une fille doit nouer des relations avec un homme. Ils s'opposent donc fermement, pour des raisons à la fois religieuses et coutumières, à toutes formes de relations amoureuses ne visant pas à terme le mariage et considèrent comme inacceptables toute relation sexuelle avant une union officielle : "Chez nous, on te donne la fille avant que tu la touches, [...] et c'est dans le coran aussi $"^{2}$. Pourtant, dans la pratique, le parcours amoureux et sexuel des filles emprunte des logiques qui ont très peu à voir avec les modèles parentaux.

\section{Virginité avant le mariage : une notion chimérique !}

9 Toutes les filles interrogées ont eu au moins une relation amoureuse avant celle qui a conduit à leur mariage. Et quant à ce qui est des relations sexuelles, il se révèle que la virginité est loin d'être la règle absolue, même chez celles qui défendent dans leurs discours le principe de la virginité pré-conjugale, la plupart ayant eu des relations sexuelles non seulement avec des amis, "petits copains", mais aussi avec ceux qui sont devenus leurs conjoints. Les élus amoureux (amis, petits copains) des filles sont recrutés dans leur sphère de vie quotidienne, le plus souvent à l'école ou dans le quartier de résidence et les critères de choix excluent toute considération religieuse ou ethnique et se fondent essentiellement sur des sentiments amoureux décrits par les filles comme des formes d'attractions interpersonnelles. Ainsi existent, à côté d'une certaine influence familiale, des normes de conduite propres aux filles Soninké qui correspondent au modèle dominant de leur contexte de socialisation.

10 Ces choix amoureux des filles, qui sont strictement individuels, ne semblent pourtant pas échapper aux parents, même si ces derniers, selon la plupart des filles interrogées, feignent de "ne rien savoir ". Cette "politique de l'autruche» des parents, touche beaucoup plus directement les pères, les mères étant presque toujours informées, soit parce que leurs filles leur ont parlé, soit, plus rarement, parce qu'elles les ont interrogées. Ceci s'explique par la nature des relations parents-enfants dans ces familles soninké (et dans les familles d'origine ouest-africaine en général) où les pères, contrairement à leurs femmes, représentent l'autorité suprême et ne sont pas directement ou facilement « accessibles » à leurs enfants, que ce soient des filles ou des garçons.

11 Cependant, la mise au courant des mères ne modifie pas chez elles cette attitude d'indifférence consciente. Au contraire, elles s'éloignent davantage de leurs filles de peur de contribuer, à l'insu et contre leurs maris, à l'établissement chez leurs enfants d'un comportement "prohibé » qui, même s'il est socialement dominant, est jugé négativement par eux et contraire à ce qu'essaie de transmettre le modèle familial. Rares sont donc les mères qui en discutent avec leurs filles; et lorsque c'est le cas, les mères somment leurs filles d'arrêter la relation et évitent ultérieurement toutes informations à ce sujet. Une des mères que nous avons interrogées reconnaît explicitement cette attitude à propos de sa fille qui est aujourd'hui séparée : "Je savais qu'elle avait un petit copain. En ce moment, son père avait déjà complètement dit oui au cousin [...]. Pour parler franchement, je savais qu'elle avait des rapports ${ }^{4}$ avec lui parce que j'ai vu des médicaments dans son sac, mais elle ne m'a rien dit avant de faire ça. Et c'est très grave ça, vous 
voyez! [...] Moi j'ai continué par faire comme si je ne savais pas. Mais je lui disais encore beaucoup, ne fais rien avec ce jeune, sinon c'est pas bon pour Dieu, et ton père va te tuer! $»^{5}$. Ce sont d'ailleurs ces comportements "à risques", d'un point de vue social, des filles qui amènent le plus souvent les parents à brusquer leur mise en union, afin d'éviter le pire - aux yeux des parents -, que leurs filles se trouvent enceintes. La même mère le reconnaît bien : "C'est moi-même qui a pressé son père pour le mariage, sinon elle va amener la honte et la malchance dans la famille ». Les pères rejoignent sur ce point leurs femmes : "Les filles d'ici là, si tu ne la maries pas vite, elle va te foutre la honte seulement "

Mais contrairement au principe de la virginité, qui est généralement considéré comme "dépassé » et qui n'est pas respecté, la quasi-totalité des jeunes filles interrogées se refusent à vivre en union libre qui, pourtant, peut prolonger une relation amoureuse pré-maritale. En revanche, aucune d'elles ne juge négativement ce mode d'union et la plupart y voient des avantages inhérents à la vie en couple : meilleure connaissance des partenaires entre eux, apprentissage de la vie de couple et facilité de rupture. Les représentations des parents à ce propos et la véhémence avec laquelle ils l'expriment, permettent de comprendre pourquoi leurs filles ne peuvent pas envisager de vivre en union libre. S'opposant déjà à toutes relations amoureuses et sexuelles en dehors d'un cadre reconnu de mariage, on peut aisément déduire leur position concernant le concubinage: intolérable, malédiction, profanation, sacrilège, désordre, honte sont autant d'expressions qui renvoient à leurs opinions sur ce sujet. Contrairement à ce qu'on observe sur le plan des relations amoureuses et sexuelles, la position de ces filles relativement à l'union libre révèle la forte contrainte de l'éducation familiale qui délimite leur espace de liberté.

C'est pourquoi alors qu'elles considèrent l'amour comme le critère déterminant dans le choix du conjoint avec, accessoirement, une légère tendance à la mobilité sociale, ce sont, avant tout, les critères endogamiques prônés par leurs parents qui ont caractérisé leurs unions. Par exemple l'endogamie de caste a été respectée chez toutes les filles de notre échantillon ; mieux, il ressort une prédominance absolue des cousins et alliés de la famille comme cela est préconisé chez les Soninké7. Et même si les conjoints sont pour la plupart scolarisés, leur niveau d'études est globalement inférieur à celui des filles. Ils ont des statuts socio-professionnels d'ouvriers et d'employés, ce qui constitue une source de précarité des couples, d'autant que les filles elles-mêmes ayant, pour diverses raisons (manque d'encouragement et de soutien familial, pauvreté et conditions de vie défavorables), abandonné précocement leur scolarité, se retrouvent concentrées dans les métiers de services dévalorisés (femmes de ménage, gardes d'enfants non déclarées) et des professions commerciales instables (vendeuse, caissière).

\section{Implication parentale dans le processus de mise en union}

L'implication parentale (père et mère) ou du groupe familial élargi (membres $d u$ fabanka ${ }^{8}$ d'origine des parents) dans le processus d'union est sans doute le point sur lequel la volonté des parents et celle des jeunes filles ont la plus grande difficulté à s'accorder, puisque le contenu et la forme que prendra la trajectoire matrimoniale des filles en dépendent.

Les filles perçoivent en général comme obsolète toute intervention familiale conduisant à une union contrainte. Pour ces jeunes femmes françaises, d'origine Soninké, mariées 
précocement, le choix d'un conjoint doit être individuel et basé sur des motivations et des critères personnels et en aucun cas faire l'objet de pressions ou d'obligations ni de la part des parents, ni encore moins du groupe familial élargi. Cependant, elles s'interdisent toutes et ce, malgré parfois de mauvaises expériences, d'exclure totalement leurs parents de leurs projets matrimoniaux. Même si avoir un copain ne doit en aucune manière faire l'objet d'une implication parentale, elles refusent néanmoins de se marier sans le moindre consentement de leurs parents, évoquant le grand désarroi et surtout la profonde rupture que cela entraînerait par rapport à leurs milieux familiaux. C'est pourquoi elles accordent, dans le cadre d'un mariage, un rôle récurrent aux parents : celui de "conseillers ». Notons ce discours contradictoire des filles, qui pensent l'époux idéal sur la base de sentiments amoureux et qui recherchent en même temps le consentement et la reconnaissance des parents (conseillers). En réalité, comme leurs parents, elles ont conscience que leur statut et leur identité matrimoniaux sont définis dans le jeu des relations familiales et sociales. Pour elles, aller contre les parents, c'est aussi aller contre les règles de la communauté.

Les parents, quant à eux, ne perçoivent pas de la même manière leurs places et rôles dans le processus matrimonial de leurs filles. En effet, c'est à eux que doit revenir le rôle prééminent dans toute union de leurs enfants, garçons ou filles. Ce sont eux, les parents, qui savent ce qui fera, dans ce domaine, le bonheur de leurs enfants. C'est pourquoi la plupart d'entre eux utilisent ce terme de «bénédiction de la famille » qui devrait accompagner tout le processus de mise en union de leurs enfants. Ce concept de «bénédiction » est évocateur de la force et de la profondeur avec lesquelles les parents conçoivent l'implication du groupe familial dans le processus qui doit amener à l'union de leurs filles. La bénédiction au sens où ils l'utilisent signifie, entre autres, une approbation et une validation, à la limite du "sacré », de l'union à travers ses différentes étapes non seulement par eux, mais également par les membres du groupe familial du pays d'origine : «Mes grands-frères sont là-bas; tu veux que je marie ma fille sans rien leur dire? Ils vont penser quoi de moi? moi-même en France ici, on m'appelle et on me demande toujours quand les enfants de mon frère là-bas vont se marier " ${ }^{9}$. Ce discours traduit la pression familiale provenant du pays Soninké, qui se manifeste à travers le canal des liens souvent très étroits que ces immigrants maintiennent avec leurs pays d'origine : liens financiers (envoi régulier d'argent aux membres de la famille); liens sociaux et culturels (participation aux décisions et aux activités du fabanka depuis la France).

\section{La présence des parents durant tout le processus}

Chez toutes les filles enquêtées, le choix de conjoint a été essentiellement assuré par les parents. Les tentatives personnelles de certaines filles qui, voulant contourner la domination parentale, ont essayé, comme cela leur a été inculqué, de présenter des hommes musulmans - même s'ils ne sont pas Soninké - à leurs parents ont été des échecs. Tous les conjoints, ont été directement choisis soit par les parents eux-mêmes, notamment les pères, soit ils ont (avec les parents) formulé une demande à leurs futurs beaux-parents, conformément à la procédure traditionnelle Soninké. Ces sollicitations et les engagements ultérieurs débutent parfois très tôt, dès la naissance des filles. Un père enquêté explique : "C'est le fils d'un ancien, très, très ancien, ami à la famille, depuis que mon père était jeune, ils sont comme les gens de la famille. C'est lui qui a demandé ma fille pour son fils depuis que ma fille est née; comment tu veux que je refuse ça? »10. 
18 Les premières rencontres et le processus de " socialisation amoureuse ", c'est-à-dire de connaissance mutuelle des prétendants, sont généralement organisés par les parents des filles qui mettent en œuvre diverses stratégies selon que le conjoint est déjà en France ou dans les pays d'origine: invitations fréquentes du garçon à la maison, exaltation de ses qualités auprès de la fille et même visite - " guidée », bien entendu du pays d'origine par la fille. En ce qui concerne le dernier point, les explications d'une enquêtée mettent en lumière les tactiques poussées de manipulation utilisées par les parents et permettent de comprendre jusqu'où ils sont prêts à aller pour réaliser leurs objectifs : "Quand on était là-bas, il y avait un cousin à moi qui était tout le temps avec nous. Il était très sympa; il nous emmenait partout, mon père et moi, il m'apprenait la langue [...] Quelques mois après mes parents ont commencé par me dire qu'il demandait tout le temps d'après moi et on m'a proposé de l'épouser " ${ }^{11}$.

19 Un autre facteur permettant de se rendre compte de la présence familiale dans l'union des jeunes filles françaises d'origine Soninké mariées précocement est que le processus matrimonial respecte, tout au moins dans sa forme, c'est-à-dire en ce qui concerne les pratiques socio-culturelles, les règles Soninké. La quasi-totalité des unions ont été sanctionnées par des fêtes et des moments de réjouissances regroupant les parents, les amis, bref, les membres de la communauté. En France, on loue des salles, on invite des artistes Soninké, etc. Selon les parents enquêtés eux-mêmes, l'initiative de ces fêtes provient des beaux-parents qui vont jusqu'à l'imposer à leurs gendres. Certains parents y apportent même leurs contributions financières en affirmant que " c'est une joie quand on marie sa fille $»^{12}$. Mais si ce discours est logiquement acceptable, la célébration des fêtes révèle surtout une certaine nécessité du conformisme et du paraître chez ces immigrés tout en confirmant leur mode de détermination communautaire.

On peut distinguer dans le processus matrimonial de toutes les filles enquêtées trois étapes principales après l'identification du conjoint, quels que soient le lieu et le mode de déroulement du processus : la rencontre ou la concertation des deux familles, la remise de la dot, aussi symbolique soit-elle, surtout lorsque le conjoint est un membre très proche de la famille, puis le mariage proprement dit (civil, religieux et traditionnel). Et le déroulement du processus ne va pas sans heurts. Ces filles qui «ne se laissent pas faire ${ }{ }^{13}$ par leurs maris ne sont pas non plus libres de toute pression exercée par leurs parents.

\section{Un parcours d'implication parentale semé d'obstacles}

21 Les filles, aussi bien que leurs parents, ne taisent pas les tractations, les antagonismes et les violences qui ont caractérisé le processus matrimonial, car les parents développent, pour arriver à leurs fins, différents stratagèmes pouvant aller de la persuasion à la contrainte absolue. Il faut préciser qu'en s'impliquant dans le processus matrimonial de leurs filles, l'objectif des parents - et cela, ils ne le dissimulent guère est de reproduire le mieux possible le modèle Soninké. Et c'est ce qui constitue la principale source de controverses, les enfants ne voyant pas les choses du même œil que leurs parents et n'acceptant ni immédiatement, ni sans réticence, les conjoints proposés par leurs parents. Toutes déclarent s'y être d'abord opposées d'une manière ou d'une autre avec plus ou moins de fermeté. C'est ce qui donne lieu aux diverses formes de négociations et d'interactions familiales qui prennent deux formes, la persuasion et la contrainte. 

fille : le père peut se révéler par exemple beaucoup plus gentil et plus prévenant envers sa fille, la mère emboîtant le pas à son mari, aussi bien avant l'annonce du projet matrimonial à la fille que pendant la phase de négociation proprement dite. Ce comportement, en plus de son rôle fonctionnel de négociation, sert donc à " préparer le terrain ». Une de nos enquêtées explique : «Mon père qui était d'habitude très distant et froid, est devenu super-sympa avec moi ; il me félicitait pour la moindre chose, me qualifiait de courageuse parce que je réussissais à l'école, de digne fille de ses parents, et tout ça; et ma mère aussi. Mais je me doutais de rien. C'est après que j'ai su que c'était pour me proposer un mec $»^{14}$. La persuasion peut également prendre la forme de fréquentes discussions, initiées par les parents, avec la fille. différentes tentatives de persuasion, ou bien lorsque la fille présente ouvertement des signes de résistance, par exemple, le refus catégorique de discuter avec les parents. Là également, tous les moyens sont bons pourvu qu'ils permettent l'affaiblissement et la déstabilisation souhaitée par les parents : culpabilisation des filles qui sont accusées de désobéir aux lois islamiques, de donner une mauvaise image de leurs parents, aussi bien au regard de la communauté d'origine que d'accueil; violences psychologiques et verbales (injures, calomnies) véhiculant un mépris des filles. Quelques enquêtées expliquent: "Ma propre mère ne m'a pas parlée pendant une semaine. Pour mon père n'en parlons pas $\|^{15}$; «À un moment, mes parents me détestaient tellement que pour me parler ils ne me regardaient pas dans les yeux $»^{16} ;$ « Mon père m'a dit qu'il me renierait, si je refusais $»^{17}$. rigoureuse de relations sexuelles avant le mariage. Comme en pays Soninké, la règle est clairement explicitée par les parents, mais ni les garçons ni les filles ne s'y soumettent et ils ont des relations sexuelles avec leurs futurs conjoints dès leurs premières rencontres et en tout cas bien avant le mariage.

entretiens révèlent que l'initiative en revient essentiellement aux filles, les garçons étant d'autant plus réticents qu'ils appartiennent à la première génération d'immigration et qu'ils ne veulent pas décevoir leurs beaux-parents. Le croisement des discours d'un père et d'une fille témoigne bien de cette réalité dont les filles ne semblent d'ailleurs pas en faire un tabou : "Moi je ne pouvais pas attendre [...] lui il faisait son timide au départ. Il parlait religion, il craignait mon père... $»^{18}$. Et le père $:$ "Nos filles d'ici, là, elles sont gâtées! Elles obligent les garçons à coucher! ${ }^{19}$. Cependant, les pères et les fiancés sont assez hypocrites, car même dans les pays d'origine, les garçons ont, la plupart du temps, des relations sexuelles avant les unions, le non-respect du principe islamique de la virginité pré-conjugale étant socialement beaucoup plus toléré pour eux.

Les parents pour qui ces transgressions de leurs filles apparaissent comme un sacrilège, se voient obligés dans la plupart des cas de précipiter la célébration de l'union dans sa totalité ou de procéder au mariage religieux afin d'en officialiser la consommation, le mariage civil et la remise de la dot par le conjoint n'intervenant qu'après. Une de nos enquêtées mariée à 17 ans déclare par exemple que son mariage a été précipité parce qu' " elle était tombée enceinte après les fiançailles".

Si le bouleversement du processus matrimonial des filles Soninké mariées jeunes en France donne lieu à des comportements parentaux coercitifs, c'est parce que le modèle socio-culturel soninké se durcit sous l'effet de la situation migratoire 


\section{Les parents : un double espace de pression communautaire d'origine soninké}

28 À bien des égards, que ce soit leurs discours systématiques relativement à la virginité pré-matrimoniale ou leur position par rapport à l'implication familiale, les parents font référence au pays d'origine. Mais il serait réducteur d'y voir la simple reproduction du modèle du pays d'origine.

Dans le contexte migratoire, ces parents subissent une double pression du groupe familial et de la communauté d'origine en général, aussi bien en France que dans les pays d'origine. C'est ce qui explique le raidissement de leur position: les parents ne reculent devant aucune difficulté pour s'impliquer dans le choix du conjoint de leurs filles et tiennent à ce que le processus matrimonial obéisse, même si c'est dans la forme, aux usages Soninké. Tout se passe comme si ces émigrés avaient continuellement besoin de prouver leur capacité à rester fidèles à leur culture et à leurs groupes d'origine.

Parlant spécifiquement du pays Soninké, Sy (1996) a, par exemple, bien expliqué que la maison paternelle (fabanka) demeure la fierté du Soninké et que s'il émigre, c'est pour en éviter l'écroulement en la soutenant contre la disette et la faim. Or le maintien du fabanka passe également et pour beaucoup, par le respect des règles matrimoniales favorisant sa reproduction, même si les enfants qui se marient n'appartiennent qu'à distance au fabanka. C'est pourquoi nos entretiens montrent que même des filles nées en France étaient demandées en mariage en tant que membres de leurs maisons paternelles depuis les pays d'origine et ce, parfois dès leur naissance.

31 Les parents ont donc tout intérêt à veiller à ce que la dignité et la fierté que leur procure en amont le soutien financier qu'ils apportent à leurs familles des pays d'origine, ne soient pas, en aval, battues en brèche par les mésalliances de leurs filles.

Dans le pays d'accueil, le mode de vie communautaire de ces immigrants Soninké (Quiminal, 1991 ; Barou, 1993 ; Poiret, 1995 ; Timera, 1996) exerce également, à travers sa fonction de maintien et de transmission, entre autres, des valeurs matrimoniales des pays d'origine, un effet de contrôle social sur les individus. Les parents se sentent, d'une manière ou d'une autre, tenus par les normes et pratiques Soninké. II y va de la perpétuation des liens avec la famille et la communauté d'origine et pour beaucoup, de leur statut. Et en sachant combien ces immigrants défendent en général leur identité d'origine, on peut aisément comprendre pourquoi ces parents sont prêts à tout pour que le choix des conjoints de leurs filles témoigne de cette fidélité aux mœurs et exigences socio-culturelles Soninké.

La désignation quasi-systématique des enfants par « filles d'ici », renvoie au concept de "deuxième génération » qui n'est pas conforme aux représentations du lignage chez les Soninké. Ce qui signifie que ces parents ont un tant soit peu compris que leurs familles, à cause de la situation migratoire, ne seront plus des espaces fermés où pourraient se reproduire à l'identique les cultures d'origine, mais plutôt des lieux de coexistence de différences culturelles. Or, les Soninké des pays d'origine, qui ne peuvent pas comprendre cette réalité, attendent et exigent toujours de ces immigrants des marques de fidélité. Se définissant déjà eux-mêmes comme Soninké avant toute 
chose, ils sont contraints de ne pas trahir cette identité, réduisant ainsi leur tolérance et leur ouverture.

Les filles mariées précocement se sont donc retrouvées prises dans des enjeux familiaux, identitaires, sociaux et économiques, surdéterminés par la logique de l'opposition entre le reniement et la fidélité, c'est-à-dire entre la soumission aux valeurs occidentales et l'affirmation des normes et valeurs Soninké. Ces tensions, du fait même de l'immigration, sont vécues par leurs parents avec intensité face à laquelle n'ont pas pu résister les effets de leur socialisation en France (Belhadj, 1998).

\section{Le processus matrimonial, source de vulnérabilité}

Si le modèle de nuptialité des jeunes filles françaises d'origine Soninké mariées précocement est proche de celui du pays d'origine, il l'est surtout du fait de l'implication familiale et de la nature endogamique du mariage.

En revanche, le processus qui conduit à l'union, de par les différentes interactions qui le déterminent, s'en différencie totalement. En effet, alors que le modèle du pays d'origine est caractérisé par leurs règles socio-culturelles d'union (prééminence du rôle familial, virginité avant le mariage), celui des filles nées et élevées en France révèle plutôt différents types de contraintes et de transgressions opposant parents et filles, les uns se pliant à la volonté des autres dans une dynamique d'antagonismes. Et c'est de cette dynamique d'antagonismes que provient principalement la vulnérabilité de chacun des acteurs impliqués dans le processus matrimonial. En effet, pour les parents, "l'effet pervers" du contexte migratoire réside dans le fait que le contrôle socioculturel des comportements de leurs enfants, assuré en règle générale par la communauté dans les pays d'origine, n'est pas réalisable en France. Au contraire, dans le pays d'accueil cette influence communautaire, qui, en général là-bas, renforce et soutient le rôle parental, se transforme, nous l'avons vu, en un dispositif de contrôle et d'évaluation des parents quant à leur capacité à prouver leur attachement à leur culture d'origine à travers le mariage de leurs filles. Il en résulte une pression qui est la principale source de vulnérabilité de chacun des acteurs impliqués, notamment les parents et leurs filles; cette vulnérabilité entraînant d'autres situations de risques.

Pour les parents immigrants, la vulnérabilité vient, avant tout, de l'existence d'un risque permanent d'échec du projet, c'est-à-dire de la possibilité concrète, dans le contexte migratoire, du refus du projet d'union par les filles ou de sa remise en cause par diverses institutions de médiation. Les filles, quant à elles, même si elles savent très bien que leur union n'est pas valorisée dans la société française, ont conscience du coût affectif et psychologique qu'aura sa remise en cause : rupture avec leurs parents et isolement auquel l'éducation parentale ne les a pas préparées.

La conjugaison des réponses apportées par chacun de ces acteurs pour pallier leurs vulnérabilités, si elles se révèlent efficaces, au moins à court terme, aussi bien pour les uns que pour les autres, engendre d'autres vulnérabilités. Les parents, de la persuasion à la culpabilisation, mettent tout en œuvre pour que le projet d'union, au moins dans sa forme, se concrétise suivant leur modèle de référence; de leur côté, les filles, ne pouvant s'opposer radicalement à la volonté parentale, se soumettent en transgressant. Tout ceci fabrique de manière continue des individus vulnérables à plusieurs points de vue : des parents vulnérables parce qu'ils se sont vus obligés d'être des pères et des mères Soninké dans le cadre du mariage de leurs filles françaises en France; des filles 
françaises vulnérables parce qu'elles ont été obligées de « redevenir » lors de leur mise en union en France, des enfants Soninké. La vulnérabilité résulte ainsi directement de la dégradation des relations intra-familiales.

Mais celle-ci s'inscrit dans un contexte de pauvreté. Ces parents déjà vulnérables à la pauvreté de par leur condition d'immigrés, ont dû en outre, et le plus souvent sans l'avoir planifié ou organisé, soit héberger leurs filles et leurs gendres, soit avancer ou dépenser de l'argent pour des cérémonies de mariage. Par exemple parce que les conjoints n'ont pas eu le temps nécessaire d'économiser, en travaillant, l'argent requis pour l'organisation de leur mise en union, avant que leur fille "d'ici» - selon les parents eux-mêmes - n'ait obligé son fiancé à " coucher » avec elle, où ne se retrouve enceinte, bref, avant qu'elle ne les oblige au mariage. Or le mariage, loin de compenser la pauvreté des conditions de vie, l'aggrave: compte tenu de leurs statuts socioprofessionnels, les conjoints "recrutés» par les parents, qui sont généralement immigrants de première génération, peu ou pas diplômés, sont exposés au chômage et au travail précaire. Enfin, ces couples sont vulnérables à la séparation et au divorce, notamment à cause de la différence de milieux de socialisation et de niveau d'études entre les conjoints. Ceci rend, par ricochet, vulnérables les parents qui, comme nous l'avons observé au cours de nos enquêtes, se voient obligés de recueillir leurs filles après de multiples tentatives de conciliation.

Notre matériau, purement "transversal », ne nous permet pas de suivre le devenir de ces couples: dans tous les cas cette situation peut maintenir, à terme, dans la vulnérabilité aussi bien les filles et leurs conjoints, que les parents qui vont continuer à " récolter ce qu'ils ont été amenés à semer ».

\section{BIBLIOGRAPHIE}

BAROU Jacques (1993) Les immigrés africains en France : des navigateurs au regroupement familial, Revue Française des Affaires sociales, 1, pp. 193-205.

BELHADJ Marnia (1998) Une «révolution» tranquille : stratégies professionnelles et dynamique familiale d'un groupe de femmes françaises d'origine algérienne, Thèse de doctorat de Sociologie, EHESS, Paris. Charbit Yves (2005) Vulnérabilité et Migrations. Journée d'étude CEPED/POPINTER, Paris.

CHASTANET Monique (1988) Le pays soninké, traits communs et diversité, Soonikara, 1, pp. 6-9. POIRET Christian (1995) L'immigration familiale d'Afrique noire en région Ile-de-France. Famille et habitat au cœur des mutations. Synthèse, Migrations et Études, 57.

POLLET Éric \& WINTER Grace (1971) La Société Soninké (Dyahunu, Mali), Bruxelles, Éditions de l'Institut de Sociologie.

QUIMINAL Catherine (1991) Gens d'ici, gens d'ailleurs. Migrations soninké et transformations villageoises, Paris, Christian Bourgeois. 
SY Yaya (1996) Les associations villageoises soninké en France (Leur rôle dans la dynamique associative africaine en France et le développement des villages d'origine), Thèse de doctorat de sociologie, Université René Descartes Paris V, Paris.

TIMERA Mahamet (1996) Les Soninké en France. D'une vie à l'autre, Paris, Karthala.

\section{NOTES}

1. Une des filles enquêtées : 19 ans, mariée.

2. Un des pères enquêtés : 53 ans, en retraite et marié.

3. Une des filles enquêtées, 23 ans, mariée.

4. Le terme Rapports utilisé par l'enquêtée signifient concrètement rapports sexuels.

5. Une des mères enquêtées : 45 ans, mariée.

6. Un des pères enquêtés : 51 ans et marié.

7. Pour plus de détails sur les Soninké, voir Pollet et Winter (1971) et plus récemment Timera (1996).

8. Le fabanka ou $k a$ est la structure centrale de l'organisation familiale en pays Soninké et se caractérise par une unité de résidence, de production et de consommation de ses membres. Il est patrilinéaire ou virilocale (Chastanet, 1988 ; Timera, 1996).

9. Un des pères enquêtés : 53 ans et père de deux des filles enquêtées.

10. Un des pères enquêtés : 52 ans et marié ; a une fille dans l'échantillon d'enquête.

11. Une des filles enquêtées : sa mère fait partie de l'échantillon d'enquête ; elle n'a pas épousé le prétendant en question.

12. Un des pères enquêtés : 61 ans ; a une fille dans l'échantillon d'enquête.

13. Une des filles enquêtées : 24 ans, mariée.

14. Une des filles enquêtées, 24 ans, mariée.

15. Une des filles enquêtées : 19 ans et mariée ; a ses deux parents dans l'échantillon d'enquête.

16. Une des filles enquêtées : 26 ans, séparée.

17. Une des filles enquêtées : 28 ans, mariée.

18. Une des filles enquêtées : 28 ans, mariée.

19. Un des pères enquêtés : 52 ans et marié ; a une fille dans l'échantillon d'enquête.

\section{AUTEUR}

\section{YVES ZOUNTCHÉGBÉ MAGNON}

Doctorant, Paris Descartes UMR CEPED (Paris Descartes-INED-IRD), 45 rue des saints Pères 75006 Paris. 